\title{
Exponential Stability of Primal-Dual Gradient Dynamics with Non-Strong Convexity
}

\author{
Xin Chen, $\mathrm{Na} \mathrm{Li}$
}

\begin{abstract}
This paper studies the exponential stability of primal-dual gradient dynamics (PDGD) for solving convex optimization problems where constraints are in the form of $A \boldsymbol{x}+B \boldsymbol{y}=\boldsymbol{d}$ and the objective is $\min _{\boldsymbol{x}, \boldsymbol{y}} f(\boldsymbol{x})+g(\boldsymbol{y})$ with strongly convex smooth $f$ but only convex smooth $g$. We show that when $g$ is a quadratic function or when $g$ and matrix $B$ together satisfy an inequality condition, the PDGD can achieve global exponential stability given that matrix $A$ is of full row rank. These results indicate that the PDGD is locally exponentially stable with respect to any convex smooth $g$ under a regularity condition. To prove the exponential stability, two quadratic Lyapunov functions are designed. Lastly, numerical experiments further complement the theoretical analysis.
\end{abstract}

Index Terms-Primal-dual gradient dynamics, exponential stability, non-strong convexity, Lyapunov function

\section{INTRODUCTION}

Continuous-time primal-dual gradient dynamics (PDGD) [1] is a prominent first-order method to solve constrained convex optimization problems. Due to its simple structure and scalability, PDGD has been widely used in many fields, such as wireless communication [2], [3], power grid operation [4]-[6], distributed resource allocation [7], [8], and imaging processing [9]. Theoretic analysis of the performance of PDGD, especially its convergence property, recently received considerable attention. A number of studies [10]-[12] were devoted to establish the asymptotic stability of PDGD using local convexity-concavity of the saddle point function, while this paper focuses on a stronger stability guarantee: the global exponential stability of PDGD.

Global exponential stability is highly desired in practice. On the one hand, it is necessary to have strong stability guarantee on practical dynamic systems, especially for those in critical infrastructures like power grids and telecommunication networks. On the other hand, global exponential stability implies many useful theoretic properties. For example, using explicit Euler discretization with sufficiently small step size, a continuous-time dynamics with global exponential stability can be discretized as an iterative algorithm that achieves linear convergence rate [13], [14]. In addition, for a perturbed system with bounded perturbation, its solution is proved to be ultimately bounded if the corresponding nominal system is globally exponentially stable [15].

There have been a number of efforts [16]-[21] in studying the exponential stability of PDGD. For equality constrained convex optimization, [16], [17] show that PDGD is globally

X. Chen and N. Li are with the School of Engineering and Applied Sciences, Harvard University, USA. Email: (chen_xin@g.harvard.edu, nali@seas.harvard.edu).

The work was supported by NSF 1608509, NSF CAREER 1553407, AFOSR YIP, and ARPA-E through the NODES program. exponentially stable when the objective is strongly convex and smooth. In [19], the theory of integral quadratic constraints is applied to prove the global exponential stability of a proximal primal-dual flow dynamics. Then [18] extends the global exponential stability result to the strongly convex optimization with affine inequality constraints using an augmented Lagrangian function. Besides, the local exponential stability of PDGD is established in [20], [21] by analyzing the spectral bounds of saddle matrices. As for the discrete-time counterpart, the primal-dual gradient descent algorithm solving a saddle point problem is proved to achieve linear convergence when the objective is strongly convex in the primal variables and strongly concave in the dual variables [22]. While recent work [23], [24] shows that only one of the strong convexity (concavity) condition is necessary if the primal-dual coupling is bilinear and the coupling matrix is of full rank.

In all the literatures above, a strongly convex and smooth objective is assumed for PDGD to achieve global exponential stability. However, in many applications, the objective of the optimization problem may not have strong convexity in all variables, such as resource allocation [7] and optimal network flow in power systems [5], [6]. Those problems can be generalized as formulation (1)

$$
\begin{gathered}
\min _{\boldsymbol{x} \in \mathbb{R}^{n}, \boldsymbol{y} \in \mathbb{R}^{m}} f(\boldsymbol{x})+g(\boldsymbol{y}) \\
\text { s.t. } \quad A \boldsymbol{x}+B \boldsymbol{y}=\boldsymbol{d}
\end{gathered}
$$

where $A \in \mathbb{R}^{k \times n}, B \in \mathbb{R}^{k \times m}, \boldsymbol{d} \in \mathbb{R}^{k}, f$ is strongly convex, but $g$ is only convex (e.g. affine function or missing).

Then a natural question to ask is whether PDGD can maintain global exponential stability when the objective is non-strongly convex; if not, what other conditions it requires to ensure at least the exponential convergence of some critical variables.

Contribution. This paper establishes the conditions under which PDGD can still achieve global exponential stability even though the objective is non-strongly convex. Specifically, we employ PDGD to solve a class of convex optimization problems in the form of (1), where $f$ is strongly convex smooth but $g$ is only convex smooth. Given that matrix $A$ is of full row rank, it is proved that PDGD is globally exponentially stable when $g$ is a quadratic function or satisfies an inequality condition together with matrix $B$. We further show that PDGD is locally exponentially stable for any convex $g$ under a regularity condition. Two quadratic Lyapunov functions with non-zero off-diagonal terms are designed to prove these results. Lastly, we provide numerical studies to complement the analysis.

The remainder of this paper is organized as follows: Section II introduces the detailed problem and some preliminaries. In Section III, we present the main results of exponential 
stability, and Section IV provides the proof sketch for these results. Numerical demonstration is carried out in Section V and conclusions are drawn in Section VI.

Notations. Throughout this paper, we use capital letters to denote matrices, lower case letters to denote scalars, and bold lower case letters to denote column vectors, respectively. Let $\langle\cdot, \cdot\rangle$ represent Euclidean inner product, and let $\|\cdot\|$ denote Euclidean norm for vectors and spectrum norm for matrices. $\mathbb{R}^{n}$ represents $n$-dimension real number space, and $\mathbb{S}^{m \times m}$ denotes $m \times m$ symmetric matrix space. For two symmetric matrices $P_{1}$ and $P_{2}$, notation $P_{1} \succeq P_{2}$ means $P_{1}-P_{2}$ is positive semi-definite.

\section{Problem Statement AND PREliminaries}

In this section, we apply PDGD to solve a convex optimization problem (1), and present the necessary conditions for the discrete-time counterpart of PDGD to achieve linear convergence.

\section{A. Problem Statement}

This paper considers solving a class of convex optimization problems in the form of (1).

Define the Lagrangian function of problem (1) as

$$
L(\boldsymbol{x}, \boldsymbol{y}, \boldsymbol{\lambda})=f(\boldsymbol{x})+g(\boldsymbol{y})+\boldsymbol{\lambda}^{\top}(A \boldsymbol{x}+B \boldsymbol{y}-\boldsymbol{d})
$$

with dual variables $\boldsymbol{\lambda} \in \mathbb{R}^{k}$. Then PDGD (3) is used to find the saddle points of the Lagrangian function $L(\boldsymbol{x}, \boldsymbol{y}, \boldsymbol{\lambda})$

$$
\begin{aligned}
& \dot{\boldsymbol{x}}=-\eta_{x} \cdot \nabla_{\boldsymbol{x}} L(\boldsymbol{x}, \boldsymbol{y}, \boldsymbol{\lambda})=-\eta_{x} \cdot\left(\nabla f(\boldsymbol{x})+A^{\top} \boldsymbol{\lambda}\right) \\
& \dot{\boldsymbol{y}}=-\eta_{y} \cdot \nabla_{\boldsymbol{y}} L(\boldsymbol{x}, \boldsymbol{y}, \boldsymbol{\lambda})=-\eta_{y} \cdot\left(\nabla g(\boldsymbol{y})+B^{\top} \boldsymbol{\lambda}\right) \\
& \dot{\boldsymbol{\lambda}}=\eta_{\lambda} \cdot \nabla_{\boldsymbol{\lambda}} L(\boldsymbol{x}, \boldsymbol{y}, \boldsymbol{\lambda})=\eta_{\lambda} \cdot(A \boldsymbol{x}+B \boldsymbol{y}-\boldsymbol{d})
\end{aligned}
$$

where $\eta_{x}, \eta_{y}, \eta_{\lambda}>0$ are the corresponding time constants.

We consider the case when $f(\boldsymbol{x})$ is strongly convex while $g(\boldsymbol{y})$ is not and restate this condition as assumption 1.

Assumption 1. Function $f$ is twice differentiable, $\mu$-strongly convex and $\ell$-smooth $(0<\mu \leq \ell)$, i.e., for all $\boldsymbol{x}_{1}, \boldsymbol{x}_{2} \in \mathbb{R}^{n}$,

$$
\begin{aligned}
\mu\left\|\boldsymbol{x}_{1}-\boldsymbol{x}_{2}\right\|^{2} \leq\left\langle\nabla f\left(\boldsymbol{x}_{1}\right)-\nabla f\left(\boldsymbol{x}_{2}\right)\right. & \left., \boldsymbol{x}_{1}-\boldsymbol{x}_{2}\right\rangle \\
& \leq \ell\left\|\boldsymbol{x}_{1}-\boldsymbol{x}_{2}\right\|^{2}
\end{aligned}
$$

And function $g$ is twice differentiable, convex and $\rho$-smooth $(\rho \geq 0)$, i.e., for all $\boldsymbol{y}_{1}, \boldsymbol{y}_{2} \in \mathbb{R}^{m}$,

$$
0 \leq\left\langle\nabla g\left(\boldsymbol{y}_{1}\right)-\nabla g\left(\boldsymbol{y}_{2}\right), \boldsymbol{y}_{1}-\boldsymbol{y}_{2}\right\rangle \leq \rho\left\|\boldsymbol{y}_{1}-\boldsymbol{y}_{2}\right\|^{2}
$$

We further make the following two assumptions.

Assumption 2. Problem (1) has a finite optimum.

Assumption 3. Matrix $A$ is of full row rank and

$$
\kappa_{1} I \preceq A A^{\top} \preceq \kappa_{2} I
$$

for some $0<\kappa_{1} \leq \kappa_{2}$.

Note that assumption 3 is crucial for PDGD (3) to achieve global exponential stability [24], since matrix $A$ is the key connection between $\boldsymbol{x}, \boldsymbol{\lambda}$ and $B \boldsymbol{y}$. By checking the KKT conditions of problem (1), we have the following proposition.

Proposition 1. Under assumption 1 and 2, any equilibrium point $\left(\boldsymbol{x}^{*}, \boldsymbol{y}^{*}, \boldsymbol{\lambda}^{*}\right)$ of the primal-dual gradient dynamics (3) is an optimal solution of problem (1).

\section{B. Necessary Conditions}

Suppose that $\boldsymbol{x}$ is the critical decision variables that we focus on, and we aim to find out the conditions ensuring the exponential convergence of $\boldsymbol{x}$. To develop some intuitions for this problem, we consider the discrete-time counterpart (6) of PDGD (3) as follows

$$
\begin{aligned}
& \boldsymbol{x}_{i+1}=\boldsymbol{x}_{i}-\nu_{x} \cdot\left(\nabla f\left(\boldsymbol{x}_{i}\right)+A^{\top} \boldsymbol{\lambda}_{i}\right) \\
& \boldsymbol{y}_{i+1}=\boldsymbol{y}_{i}-\nu_{y} \cdot\left(\nabla g\left(\boldsymbol{y}_{i}\right)+B^{\top} \boldsymbol{\lambda}_{i}\right) \\
& \boldsymbol{\lambda}_{i+1}=\boldsymbol{\lambda}_{i}+\nu_{\lambda} \cdot\left(A \boldsymbol{x}_{i}+B \boldsymbol{y}_{i}-\boldsymbol{d}\right)
\end{aligned}
$$

where $i$ denotes the iteration number, and $\nu_{x}, \nu_{y}, \nu_{\lambda}>0$ are the corresponding step sizes.

Then the following proposition shows the convergence synchronicity of different variables and implies the necessary conditions for achieving linear convergence rate of $\boldsymbol{x}$.

Proposition 2. For the primal-dual gradient algorithm (6), suppose that $\left\{\boldsymbol{x}_{i}\right\}$ achieves linear convergence rate, in the sense that, there exists $c_{x} \geq 0$ and $\vartheta \in(0,1)$ such that

$$
\left\|\boldsymbol{x}_{i}-\boldsymbol{x}^{*}\right\| \leq c_{x} \cdot \vartheta^{i}
$$

Then $\left\{\boldsymbol{y}_{i}\right\}$ and $\left\{\boldsymbol{\lambda}_{i}\right\}$ also achieve linear convergence rate, in the sense that there exist constants $c_{\lambda}, c_{y}, c_{g} \geq 0$ such that

$$
\begin{aligned}
\left\|\boldsymbol{\lambda}_{i}-\boldsymbol{\lambda}^{*}\right\| & \leq c_{\lambda} \cdot \vartheta^{i} \\
\left\|B\left(\boldsymbol{y}_{i}-\boldsymbol{y}^{*}\right)\right\| & \leq c_{y} \cdot \vartheta^{i} \\
\left\|B\left(\nabla g\left(\boldsymbol{y}_{i}\right)-\nabla g\left(\boldsymbol{y}^{*}\right)\right)\right\| & \leq c_{g} \cdot \vartheta^{i}
\end{aligned}
$$

The proof of proposition 2 is provided in Appendix VI-H. Proposition 2 indicates that the convergence of different variables is not separate but exhibits synchronicity, hence it is suggested to analyze the the exponential stability of PDGD (3) in all variables simultaneously.

\section{MAIN RESUltS}

In this section, we consider the quadratic case and the general case of $g(\boldsymbol{y})$, and present the global (local) exponential stability results of PDGD (3).

For explicit expression, we stack $\boldsymbol{x}, \boldsymbol{y}, \boldsymbol{\lambda}$ into vector $\boldsymbol{z}:=$ $\left[\boldsymbol{x}^{\top}, \boldsymbol{y}^{\top}, \boldsymbol{\lambda}^{\top}\right]^{\top}$ and define $\boldsymbol{z}^{*}:=\left[\boldsymbol{x}^{* \top}, \boldsymbol{y}^{* \top}, \boldsymbol{\lambda}^{* \top}\right]^{\top}$ as one of the equilibrium points of PGDG (3).

\section{A. Quadratic Case of $g(y)$}

Consider the quadratic case when $g(\boldsymbol{y})$ is a quadratic function given by

$$
g(\boldsymbol{y})=\frac{1}{2} \boldsymbol{y}^{\top} G \boldsymbol{y}+\boldsymbol{g}^{\top} \boldsymbol{y}+g_{0}
$$

with $g_{0} \in \mathbb{R}, \boldsymbol{g} \in \mathbb{R}^{m}, G \in \mathbb{S}^{m \times m}$. By assumption 1, we have $0 \preceq G \preceq \rho I$.

For this case, the equilibrium point set $\Psi$ of PDGD (3) is specified by proposition 3 , and its global exponential stability is stated as theorem 1 .

Proposition 3. Under assumption 1, 2 and 3, when $g(\boldsymbol{y})$ is a quadratic function in the form of (9), the equilibrium point set $\Psi$ of the primal-dual gradient dynamics (3) is given by

$$
\Psi:=\left\{\hat{\boldsymbol{z}} \mid \hat{\boldsymbol{x}}=\boldsymbol{x}^{*}, \hat{\boldsymbol{\lambda}}=\boldsymbol{\lambda}^{*}, B \hat{\boldsymbol{y}}=B \boldsymbol{y}^{*}, G \hat{\boldsymbol{y}}=G \boldsymbol{y}^{*}\right\}
$$


See Appendix VI-G for the proof of proposition 3.

Proposition 3 implies that the components $\boldsymbol{x}^{*}$ and $\boldsymbol{\lambda}^{*}$ of the equilibrium points are unique, while $\boldsymbol{y}^{*}$ is non-unique and $\hat{\boldsymbol{y}}-\boldsymbol{y}^{*} \in \operatorname{ker}(B) \cap \operatorname{ker}(G)$ for any $\hat{\boldsymbol{z}} \in \Psi$.

Theorem 1. Under assumption 1, 2 and 3, when $g(\boldsymbol{y})$ is a quadratic function in the form of (9), the prime-dual gradient dynamics (3) is globally exponentially stable in the sense that, there exist constants $a_{x}, a_{\lambda}, a_{y_{B}}, a_{y_{G}} \geq 0$ and $\tau>0$ such that

$$
\begin{aligned}
\left\|\boldsymbol{x}(t)-\boldsymbol{x}^{*}\right\| & \leq a_{x} \cdot e^{-\tau t} \\
\left\|\boldsymbol{\lambda}(t)-\boldsymbol{\lambda}^{*}\right\| & \leq a_{\lambda} \cdot e^{-\tau t} \\
\left\|B\left(\boldsymbol{y}(t)-\boldsymbol{y}^{*}\right)\right\| & \leq a_{y_{B}} \cdot e^{-\tau t} \\
\left\|G\left(\boldsymbol{y}(t)-\boldsymbol{y}^{*}\right)\right\| & \leq a_{y_{G}} \cdot e^{-\tau t}
\end{aligned}
$$

The proof of theorem 1 is provided in Section IV-A. Equation (11) indicates that the distance between the solution $\boldsymbol{z}(t)$ and $\Psi$ converges to zero exponentially.

Remark 1: In theorem 1, we only take $g(\boldsymbol{y})$ as quadratic function, while $f(\boldsymbol{x})$ can be any strongly convex function. In this case, PDGD (3) is not necessarily a linear time-invariant system, but always preserves global exponential stability.

\section{B. General Case of $g(y)$}

Now consider a general convex function $g(\boldsymbol{y})$. Since it is not easy to analyze the global exponential stability of PDGD (3) with an unclear equilibrium point set of $\boldsymbol{y}^{*}$, we supplement assumption 4 to make optimal $\boldsymbol{y}^{*}$ to be unique. An intuition for assumption 4 is that if $B$ is the all-zero matrix, assumption 4 reduces to the condition that $g(\boldsymbol{y})$ is strongly convex. Actually, assumption 4 "mimics" a strong convexity condition in $\boldsymbol{y}$ and use matrix $B$ to make up for the strong convexity deficit of $g(\boldsymbol{y})$.

Assumption 4. For any $\boldsymbol{y}_{1}, \boldsymbol{y}_{2} \in \mathbb{R}^{m}$, there exists constant $\gamma>0$ such that

$$
\begin{aligned}
& \left(\boldsymbol{y}_{1}-\boldsymbol{y}_{2}\right)^{\top} B^{\top} B\left(\boldsymbol{y}_{1}-\boldsymbol{y}_{2}\right) \\
& \quad+\left\langle\nabla g\left(\boldsymbol{y}_{1}\right)-\nabla g\left(\boldsymbol{y}_{2}\right), \boldsymbol{y}_{1}-\boldsymbol{y}_{2}\right\rangle \geq \gamma \cdot\left\|\boldsymbol{y}_{1}-\boldsymbol{y}_{2}\right\|^{2}
\end{aligned}
$$

Proposition 4. Under assumption 1, 2, 3, 4, the prime-dual gradient dynamics (3) has a unique equilibrium point $\boldsymbol{z}^{*}$.

See Appendix VI-G for the proof of proposition 4. Noted that assumption 4 is a sufficient condition for the uniqueness of $z^{*}$ but not necessary. For example, when $B$ is the all-zero matrix and $g(\boldsymbol{y}):=\sum_{i=1}^{m} y_{i}^{4}$, assumption 4 does not hold, while $\boldsymbol{z}^{*}$ is unique with $\boldsymbol{y}^{*}=\mathbf{0}$.

Accordingly, the global exponential stability of PDGD is established as the following theorem.

Theorem 2. Under assumption 1, 2, 3 and 4, the prime-dual gradient dynamics (3) is globally exponentially stable, in the sense that, there exist constants $c_{z} \geq 0$ and $\tau>0$ such that

$$
\left\|\boldsymbol{z}(t)-\boldsymbol{z}^{*}\right\| \leq c_{z} \cdot e^{-\tau t}
$$

The proof of theorem 2 is provided in Section IV-B.

One special case of assumption 4 is that $B^{\top} B \succeq \gamma I$ for some $\gamma>0$, i.e., $B^{\top} B$ is positive definite. This is equivalent to the condition that matrix $B$ is of full column rank. Thus we have the following corollary.

Corollary 1. Under assumption 1, 2 and 3, if matrix $B$ is of full column rank, the prime-dual gradient dynamics (3) is globally exponentially stable in the sense of (13).

\section{Local Exponential Convergence}

For a finite-dimension nonlinear system, it is well-known that if the linearized system based on an equilibrium point is exponentially stable, then the original system is locally exponentially stable around this equilibrium point. Moreover, under assumption 1 and 2, it proves that PDGD (3) globally asymptotically converges to one of the equilibrium points in $\Psi$ [5], [10]. Inspired by those facts and the quadratic case, we claim the local exponential convergence of PDGD (3) with the following theorem.

Theorem 3. Under assumption 1, 2 and 3, suppose that the trajectory $\boldsymbol{z}(t)$ following the prime-dual gradient dynamics (3) globally asymptotically converges to the equilibrium point $\boldsymbol{z}^{*}$, if we have

$$
B^{\top} B+\nabla^{2} g\left(\boldsymbol{y}^{*}\right) \succ 0
$$

then there exist a time $t_{\delta} \geq 0$ and constants $\tau>0$ such that for any time $t \geq t_{\delta}$,

$$
\left\|\boldsymbol{z}(t)-\boldsymbol{z}^{*}\right\| \leq\left\|\boldsymbol{z}\left(t_{\delta}\right)-\boldsymbol{z}^{*}\right\| \cdot e^{-\tau\left(t-t_{\delta}\right)}
$$

See Appendix VI-D for the proof.

\section{EXPONENTIAL STABILITY ANALYSIS}

In this section, we present the proofs for theorem 1 and 2. To begin with, we introduce the following lemma, whose proof can be found in [18, Appendix D].

Lemma 1. Under assumption 1, for any $\boldsymbol{x} \in \mathbb{R}^{n}$, there exists a symmetric matrix $F(\boldsymbol{x}) \in \mathbb{S}^{n \times n}$ that depends on $\boldsymbol{x}$ and satisfies $\mu I \preceq F(\boldsymbol{x}) \preceq \ell I$, such that

$$
\nabla f(\boldsymbol{x})-\nabla f\left(\boldsymbol{x}^{*}\right)=F(\boldsymbol{x})\left(\boldsymbol{x}-\boldsymbol{x}^{*}\right)
$$

For any $\boldsymbol{y} \in \mathbb{R}^{m}$, there exists a symmetric matrix $G(\boldsymbol{y}) \in$ $\mathbb{S}^{m \times m}$ that depends on $\boldsymbol{y}$ and satisfies $0 \preceq G(\boldsymbol{y}) \preceq \rho I$, such that

$$
\nabla g(\boldsymbol{y})-\nabla g\left(\boldsymbol{y}^{*}\right)=G(\boldsymbol{y})\left(\boldsymbol{y}-\boldsymbol{y}^{*}\right)
$$

\section{A. Proof of Theorem 1}

For matrix $G$, pick up $\left\{\sigma_{i}, \boldsymbol{u}_{i}\right\}_{i=1,2, \cdots, m}$ as its eigen-pairs and satisfying the following three properties:

(1) $\left\{\boldsymbol{u}_{i}\right\}_{i=1,2, \cdots, m}$ form an orthonormal basis of $\mathbb{R}^{m}$.

(2) The first $l$ eigenvalues are positive, i.e. $\sigma_{1}, \sigma_{2} \cdots, \sigma_{l}>0$ and $\sigma_{l+1}, \sigma_{l+2}, \cdots, \sigma_{m}=0$.

(3) $\operatorname{ker}(G) \cap \operatorname{ker}(B)=\operatorname{span}\left(\boldsymbol{u}_{l+r+1}, \boldsymbol{u}_{l+r+2}, \cdots, \boldsymbol{u}_{m}\right)$ for a certain $r \in\{0,1, \cdots, m-l\}$, where $r=m-l$ means $\operatorname{ker}(G) \cap \operatorname{ker}(B)=\{\mathbf{0}\}$.

It can be checked that such eigen-pairs $\left\{\sigma_{i}, \boldsymbol{u}_{i}\right\}$ always exist. Define matrix $U \in \mathbb{R}^{m \times(l+r)}$ as

$$
U:=\left[\boldsymbol{u}_{1}, \boldsymbol{u}_{2}, \cdots, \boldsymbol{u}_{l+r}\right]
$$


which collects all the eigenvectors of $G$ except those in the space $\operatorname{ker}(G) \cap \operatorname{ker}(B)$. Then matrix $G$ can be rewritten as $G=U \Sigma U^{\top}$ with $\Sigma:=\operatorname{diag}\left(\sigma_{1}, \sigma_{2}, \cdots, \sigma_{l+r}\right)$. Since we have $\operatorname{row}(B) \subseteq \operatorname{Col}(U)$ by definition, there exists a matrix $T \in \mathbb{R}^{(l+r) \times k}$ such that $B^{\top}=U T$, i.e., $B=T^{\top} U^{\top}$.

To prove theorem 1, we design the quadratic Lyapunov function $V_{1}(\boldsymbol{z})$ as

$$
V_{1}(\boldsymbol{z})=\left(\boldsymbol{z}-\boldsymbol{z}^{*}\right)^{\top} P_{1}\left(\boldsymbol{z}-\boldsymbol{z}^{*}\right)
$$

where $P_{1} \in \mathbb{R}^{(m+n+k) \times(m+n+k)}$ is defined by

$$
P_{1}=\left[\begin{array}{ccc}
\frac{\alpha}{\eta_{x}} I & 0 & \frac{1}{\eta_{\lambda}} A^{\top} \\
0 & \frac{\alpha}{\eta_{y}} U U^{\top} & -\frac{\beta}{\eta_{\lambda}} B^{\top} \\
\frac{1}{\eta_{\lambda}} A & -\frac{\beta}{\eta_{\lambda}} B & \frac{\alpha}{\eta_{\lambda}} I
\end{array}\right]
$$

Here, parameter $\alpha$ is a sufficiently large positive number, and parameter $\beta$ is a sufficiently small positive number.

Lemma 2. $P_{1}$ is positive semidefinite. $V_{1}(\boldsymbol{z})=0$ if and only if $\boldsymbol{x}=\boldsymbol{x}^{*}, \boldsymbol{\lambda}=\boldsymbol{\lambda}^{*}, B \boldsymbol{y}=B \boldsymbol{y}^{*}$ and $G \boldsymbol{y}=G \boldsymbol{y}^{*}$.

See Appendix VI-E for the proof of lemma 2.

If we can show that the time derivative of $V_{1}(\boldsymbol{z})$ along the trajectory of PDGD (3) satisfies

$$
\frac{d V_{1}(\boldsymbol{z})}{d t} \leq-\tau V_{1}(\boldsymbol{z})
$$

for $\tau=\frac{\beta^{2}}{\alpha}>0$, then theorem 1 is proved. The following part is devoted to prove the property (18).

With lemma 1, PDGD (3) can be equivalently rewritten as

$$
\begin{aligned}
\frac{d \boldsymbol{z}}{d t}= & {\left[\begin{array}{cc}
-\eta_{x}\left(\nabla_{\boldsymbol{x}} L(\boldsymbol{x}, \boldsymbol{y}, \boldsymbol{\lambda})-\nabla_{\boldsymbol{x}} L\left(\boldsymbol{x}^{*}, \boldsymbol{y}^{*}, \boldsymbol{\lambda}^{*}\right)\right) \\
-\eta_{y}\left(\nabla_{\boldsymbol{y}} L(\boldsymbol{x}, \boldsymbol{y}, \boldsymbol{\lambda})-\nabla_{\boldsymbol{y}} L\left(\boldsymbol{x}^{*}, \boldsymbol{y}^{*}, \boldsymbol{\lambda}^{*}\right)\right) \\
\eta_{\lambda}\left(\nabla_{\boldsymbol{\lambda}} L(\boldsymbol{x}, \boldsymbol{y}, \boldsymbol{\lambda})-\nabla_{\boldsymbol{\lambda}} L\left(\boldsymbol{x}^{*}, \boldsymbol{y}^{*}, \boldsymbol{\lambda}^{*}\right)\right)
\end{array}\right] } \\
= & \underbrace{\left[\begin{array}{ccc}
-\eta_{x} F(\boldsymbol{x}) & 0 & -\eta_{x} A^{\top} \\
0 & -\eta_{y} G(\boldsymbol{y}) & -\eta_{y} B^{\top} \\
\eta_{\lambda} A & \eta_{\lambda} B & 0
\end{array}\right]}_{:=W(\boldsymbol{z})}\left(\boldsymbol{z}-\boldsymbol{z}^{*}\right)
\end{aligned}
$$

Here, since $g(\boldsymbol{y})$ is a quadratic function in the form of (9), we have $G(\boldsymbol{y}) \equiv G$.

Then $\frac{d V_{1}(\boldsymbol{z})}{d t}$ can be formulated as

$$
\begin{aligned}
& \frac{d V_{1}(\boldsymbol{z})}{d t}=\dot{\boldsymbol{z}}^{\top} P_{1}\left(\boldsymbol{z}-\boldsymbol{z}^{*}\right)+\left(\boldsymbol{z}-\boldsymbol{z}^{*}\right)^{\top} P_{1} \dot{\boldsymbol{z}} \\
& \quad=\left(\boldsymbol{z}-\boldsymbol{z}^{*}\right)^{\top}\left[W(\boldsymbol{z})^{\top} P_{1}+P_{1} W(\boldsymbol{z})\right]\left(\boldsymbol{z}-\boldsymbol{z}^{*}\right)
\end{aligned}
$$

Hence, it is sufficient to show property (18) by the following lemma. See Appendix VI-A for the proof of lemma 3.

Lemma 3. For any $z \in \mathbb{R}^{n+m+k}$, we have

$$
W(\boldsymbol{z})^{\top} P_{1}+P_{1} W(\boldsymbol{z}) \preceq-\tau P_{1}
$$

In this way, we prove theorem 1 using (18) and lemma 3.

\section{B. Proof of Theorem 2}

To prove theorem 2, we design the quadratic Lyapunov function $V_{2}(\boldsymbol{z})$ as

$$
V_{2}(\boldsymbol{z})=\left(\boldsymbol{z}-\boldsymbol{z}^{*}\right)^{\top} P_{2}\left(\boldsymbol{z}-\boldsymbol{z}^{*}\right)
$$

where $P_{2} \in \mathbb{R}^{(m+n+k) \times(m+n+k)}$ is defined by

$$
P_{2}=\left[\begin{array}{ccc}
\frac{\alpha}{\eta_{x}} I & 0 & \frac{1}{\eta_{\lambda}} A^{\top} \\
0 & \frac{\alpha}{\eta_{y}} I & -\frac{\beta}{\eta_{\lambda}} B^{\top} \\
\frac{1}{\eta_{\lambda}} A & -\frac{\beta}{\eta_{\lambda}} B & \frac{\alpha}{\eta_{\lambda}} I
\end{array}\right]
$$

Here, parameter $\alpha$ is a sufficiently large positive number, and parameter $\beta$ is a sufficiently small positive number.

Lemma 4. $P_{2}$ is positive definite. $V_{2}(\boldsymbol{z})=0$ if and only if $z=z^{*}$.

See Appendix VI-F for the proof of lemma 4.

Similar to (18), if we can prove that the time derivative of $V_{2}(\boldsymbol{z})$ along the trajectory of PDGD (3) satisfies

$$
\frac{d V_{2}(\boldsymbol{z})}{d t} \leq-\tau V_{2}(\boldsymbol{z})
$$

for $\tau=\frac{\beta^{2}}{\alpha}>0$, then theorem 2 is proved. To show property (24), it is sufficient to prove lemma 5. See Appendix VI-B for its proof.

Lemma 5. For any $z \in \mathbb{R}^{n+m+k}$, we have

$$
W(\boldsymbol{z})^{\top} P_{2}+P_{2} W(\boldsymbol{z}) \preceq-\tau P_{2}
$$

By lemma 5 and (24), we prove theorem 2.

\section{NUMERICAL EXAMPLES}

\section{A. Quadratic Case of $g(y)$}

For problem (1), let $n=60, m=50$ and $k=20$. The time constant is set as $\eta=\eta_{x}=\eta_{y}=\eta_{\lambda}$. Define $f(\boldsymbol{x})=\frac{1}{2} \boldsymbol{x}^{\top} F \boldsymbol{x}$ where $F:=5 I+F_{0}^{\top} F_{0}$ and $F_{0}$ is a $n \times n$ random matrix. Define $g(\boldsymbol{y})=\frac{1}{2} \boldsymbol{y}^{\top} G \boldsymbol{y}$ where $G:=\operatorname{diag}\left(0, G_{0}^{\top} G_{0}\right)$ and $G_{0}$ is a $r_{G} \times(m-1)$ random matrix with $r_{G}=40$; then $G$ is a positive semi-definite matrix with the rank at most $r_{G}$. Let $B:=\left[\mathbf{0}, B_{0}\right]$ and $B_{0}$ is a $k \times(m-1)$ random matrix. Under this setting, we have $\boldsymbol{e}_{1} \in \operatorname{ker}(G) \cap \operatorname{ker}(B)$ where $\boldsymbol{e}_{1}=[1,0, \cdots, 0]^{\top}$. $A$ and $\boldsymbol{b}$ are also random matrix and random vector respectively.
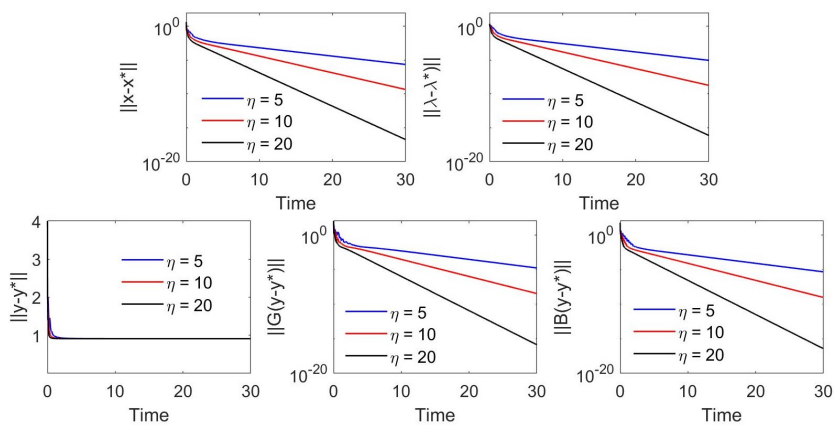

Fig. 1. Convergence results of PDGD with different time constants when $g(\boldsymbol{y})$ is a quadratic function.

We pick up an arbitrary equilibrium point in $\Psi$ as $z^{*}$, then the convergence results of PDGD are shown as figure 1 . It is observed that $\left\|\boldsymbol{x}-\boldsymbol{x}^{*}\right\|,\left\|\boldsymbol{\lambda}-\boldsymbol{\lambda}^{*}\right\|,\left\|B\left(\boldsymbol{y}-\boldsymbol{y}^{*}\right)\right\|$ and $\left\|G\left(\boldsymbol{y}-\boldsymbol{y}^{*}\right)\right\|$ converge exponentially to zero while $\left\|\boldsymbol{y}-\boldsymbol{y}^{*}\right\|$ does not, which follows the statements in theorem 1 . 


\section{B. General Case of $g(y)$}

In this case, let $g(\boldsymbol{y})=\sum_{i=1}^{m} y_{i}^{4}$ and $n=60, m=20$. $F_{0}, A, B, b$ are all random matrices or vector. We set $k=10$ and $k=30$ respectively, and run simulations for these two cases. The convergence results of PDGD are presented as figure 2. When $k=30$, matrix $B$ is of full column rank, thus assumption 4 holds and PDGD is globally exponentially stable. When $k=10$, assumption 4 is not satisfied, so PDGD exhibits asymptotic convergence at first, then (Time $>30$ ) exponentially converges to the equilibrium point due to the local exponential stability.
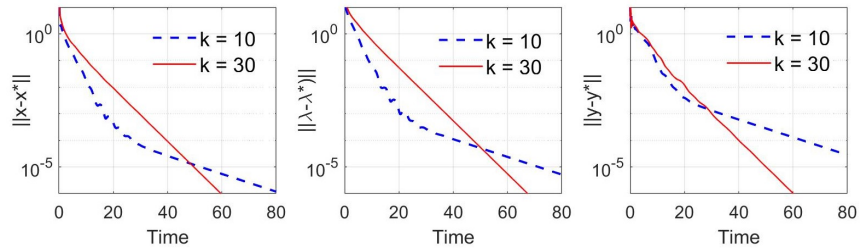

Fig. 2. Convergence results of PDGD when $g(\boldsymbol{y})=\sum_{i}^{m} y_{i}^{4}$.

\section{CONCLUSION}

In this paper, we prove that the primal-dual gradient dynamics (3) can achieve global (local) exponential stability for convex smooth optimization problems in the form of (1) with non-strong convexity in their objectives. Our main results are summarized as theorem 1, theorem 2 and theorem 3, which are proved with two quadratic Lyapunov functions. Numerical experiments are implemented and further validate these results. Future work is to extend these results to convex optimization problems with both equality and inequality constraints.

\section{REFERENCES}

[1] T. Kose, "Solutions of saddle value problems by differential equations," Econometrica, Journal of the Econometric Society, pp. 59-70, 1956.

[2] J. Chen and V. K. Lau, "Convergence analysis of saddle point problems in time varying wireless systems-control theoretical approach," IEEE Transactions on Signal Processing, vol. 60, no. 1, pp. 443-452, 2012.

[3] M. Chiang, S. H. Low, A. R. Calderbank, and J. C. Doyle, "Layering as optimization decomposition: A mathematical theory of network architectures," Proceedings of the IEEE, vol. 95, no. 1, pp. 255-312, 2007.

[4] C. Zhao, U. Topcu, N. Li, and S. Low, "Design and stability of load side primary frequency control in power systems," IEEE Trans. Autom. Control, vol. 59, no. 5, pp. 1177-1189, May 2014.

[5] N. Li, C. Zhao, and L. Chen, "Connecting automatic generation control and economic dispatch from an optimization view, IEEE Trans. Control Netw. Syst., vol. 3, no. 3, pp. 254-264, Sept. 2016.

[6] X. Chen, C. Zhao and N. Li, "Distributed automatic load-frequency control with optimality in power systems," arXiv preprint, arXiv:1811.00892, 2018.

[7] D. Ding and M. R. Jovanović, "A primal-dual laplacian gradient flow dynamics for distributed resource allocation problems," 2018 Annual American Control Conference (ACC), Milwaukee, WI, pp. 5316-5320, 2018.

[8] A. Ferragut, F. Paganini, "Network resource allocation for users with multiple connections: fairness and stability," IEEE/ACM Transactions on Networking, v.22 n.2, pp. 349-362, April 2014.

[9] A. Chambolle and T. Pock, "A first-order primal-dual algorithm for convex problems with applications to imaging," Journal of Mathematical Imaging and Vision, vol. 40, no. 1, pp. 120-145, May 2011.

[10] A. Cherukuri, E. Mallada, and J. Cortés, "Asymptotic convergence of constrained primaldual dynamics," Systems \& Control Letters, vol. 87, pp. 10-15, 2016.
[11] A. Cherukuri, E. Mallada, S. Low, and J. Cortés, "The role of convexity on saddle-point dynamics: Lyapunov function and robustness," arXiv preprint, arXiv:1608.08586, 2016.

[12] A. Cherukuri, B. Gharesifard, and J. Cortes, "Saddle-point dynamics: conditions for asymptotic stability of saddle points," SIAM J. Control Optim., vol. 55, no. 1, pp. 486-511, 2017.

[13] A. M. Stuart, "Numerical analysis of dynamical systems," Acta Numerica, vol. 3, pp. 467-572, 1994.

[14] H. J. Stetter, Analysis of Discretization Methods for Ordinary Differential Equations. Springer, vol. 23, 1973.

[15] H. K. Khalil, Nonlinear Systems, 3rd ed. NJ, USA: Prentice-Hall, 2002.

[16] S. K. Niederländer, F. Allgöwer and J. Cortés, "Exponentially fast distributed coordination for nonsmooth convex optimization," 2016 IEEE 55 th Conference on Decision and Control (CDC), Las Vegas, NV, pp. 1036-1041, 2016.

[17] J. Cortés and S. K. Niederländer, "Distributed coordination for nonsmooth convex optimization via saddle-point dynamics," Journal of Nonlinear Science, pp. 1-26, 2018.

[18] G. Qu and N. Li, "On the exponential stability of primal-dual gradient dynamics," arXiv preprint, arXiv:1803.01825, 2018.

[19] N. K. Dhingra, S. Z. Khong, and M. R. Jovanovic, "The proximal augmented Lagrangian method for nonsmooth composite optimization," IEEE Transactions on Automatic Control, 2018.

[20] M. Benzi, G. H. Golub, and J. Liesen, "Numerical solution of saddle point problems," Acta numerica, vol. 14, pp. 1-137, 2005.

[21] S.-Q. Shen, T.-Z. Huang, and J. Yu, "Eigenvalue estimates for preconditioned nonsymmetric saddle point matrices," SIAM Journal on Matrix Analysis and Applications, vol. 31, no. 5, pp. 2453-2476, 2010.

[22] P. Balamurugan, and F. Bach, "Stochastic variance reduction methods for saddle-point problems," In Advances in Neural Information Processing Systems, pp. 1416-1424. 2016.

[23] S. S. Du, J. Chen, L. Li, etc. "Stochastic variance reduction methods for policy evaluation," 34th International Conference on Machine Learning, vol. 70, pp. 1049-1058, 2017.

[24] S. S. Du, W. Hu, "Linear convergence of the primal-dual gradient method for convex-concave saddle point problems without strong convexity," arXiv preprint, arXiv:1802.01504, 2018.

\section{APPENDIX}

\section{A. Proof of Lemma 3}

Plugging $P_{1}$ (17) into equation (21) with $\tau=\frac{\beta^{2}}{\alpha}$, we obtain $Q_{1}(\boldsymbol{z}):=-W(\boldsymbol{z})^{\top} P_{1}-P_{1} W(\boldsymbol{z})-\tau P_{1}=$

$$
\left[\begin{array}{ccc}
Q_{x} & (\beta-1) A^{\top} B & \frac{\eta_{x}}{\eta_{\lambda}} F A^{\top}-\frac{\beta^{2}}{\alpha \eta_{\lambda}} A^{\top} \\
(\beta-1) B^{\top} A & Q_{y} & \frac{\beta^{3}}{\alpha \eta_{\lambda}} B^{\top}-\frac{\beta \eta_{y}}{\eta_{\lambda}} G B^{\top} \\
\frac{\eta_{x}}{\eta_{\lambda}} A F-\frac{\beta^{2}}{\eta_{\lambda} \alpha} A & \frac{\beta^{3}}{\alpha \eta_{\lambda}} B-\frac{\beta \eta_{y}}{\eta_{\lambda}} B G & Q_{\lambda}
\end{array}\right]
$$

with

$$
\begin{aligned}
Q_{x} & :=2 \alpha F-2 A^{\top} A-\frac{\beta^{2}}{\eta_{x}} I \\
Q_{y} & :=2 \alpha G+2 \beta B^{\top} B-\frac{\beta^{2}}{\eta_{y}} U U^{\top} \\
Q_{\lambda} & :=2 \frac{\eta_{x}}{\eta_{\lambda}} A A^{\top}-2 \frac{\beta \eta_{y}}{\eta_{\lambda}} B B^{\top}-\frac{\beta^{2}}{\eta_{\lambda}} I
\end{aligned}
$$

Here, we denote $F(\boldsymbol{x})$ and $G(\boldsymbol{y})$ as $F$ and $G$ respectively for notation simplification. In (26), we apply $B=T^{\top} U^{\top}$ (Section IV-A) and thus have

$$
B U U^{\top}=T^{\top} U^{\top} U U^{\top}=T^{\top} U^{\top}=B
$$

In (27b), we use $G U U^{\top}+U U^{\top} G=2 U \Sigma U^{\top}=2 G$.

To prove Lemma 3 , it is sufficient to show $Q_{1}(\boldsymbol{z}) \succeq 0$ for any $z \in \mathbb{R}^{n+m+k}$. In the follows, we will use the Schur complement argument twice to prove it. 
Denote $\omega$ as the largest eigenvalue of $B^{\top} B$, then $0 \preceq$ $B B^{\top} \preceq \omega I$ and $0 \preceq B^{\top} B \preceq \omega I$, and we have

$$
Q_{\lambda} \succeq \underbrace{\left(2 \frac{\eta_{x}}{\eta_{\lambda}} \kappa_{1}-2 \frac{\eta_{y}}{\eta_{\lambda}} \beta \omega-\frac{\beta^{2}}{\eta_{\lambda}}\right)}_{:=c_{1}(\beta)} \cdot I
$$

Firstly, consider the Schur complement ${ }^{1}$ of the block $Q_{\lambda}$ in $Q_{1}(\boldsymbol{z})$ (26), which is denoted as $\bar{Q}_{1}(\boldsymbol{z})$. Using (28), we have

$$
\bar{Q}_{1}(\boldsymbol{z}) \succeq \hat{Q}_{1}(\boldsymbol{z}):=\left[\begin{array}{cc}
Q_{x}-M_{1} & M_{2} \\
M_{2}^{\top} & Q_{y}-M_{3}
\end{array}\right]
$$

where

$$
\begin{aligned}
& M_{1}:=\frac{1}{c_{1} \eta_{\lambda}^{2}}\left(\eta_{x} F-\frac{\beta^{2}}{\alpha} I\right) A^{\top} A\left(\eta_{x} F-\frac{\beta^{2}}{\alpha} I\right) \\
& M_{2}:=(\beta-1) A^{\top} B-\frac{\beta}{c_{1} \eta_{\lambda}^{2}}\left(\eta_{x} F-\frac{\beta^{2}}{\alpha} I\right) A^{\top} B\left(\frac{\beta^{2}}{\alpha} I-\eta_{y} G\right) \\
& M_{3}:=\frac{\beta^{2}}{c_{1} \eta_{\lambda}^{2}}\left(\frac{\beta^{2}}{\alpha} I-\eta_{y} G\right) B^{\top} B\left(\frac{\beta^{2}}{\alpha} I-\eta_{y} G\right)
\end{aligned}
$$

Since $\mu I \preceq F \preceq \ell I$ and $A^{\top} A \preceq \kappa_{2} I$, we have

$$
\begin{aligned}
Q_{x} & -M_{1}=2 \alpha F-2 A^{\top} A-\frac{\beta^{2}}{\eta_{x}} I-M_{1} \\
\succeq & \underbrace{\left[2 \alpha \mu-2 \kappa_{2}-\frac{\beta^{2}}{\eta_{x}}-\frac{\kappa_{2}}{c_{1} \eta_{\lambda}^{2}}\left(\eta_{x} \ell+\frac{\beta^{2}}{\alpha}\right)^{2}\right]}_{:=c_{2}(\alpha, \beta)} \cdot I
\end{aligned}
$$

Secondly, consider the Schur complement of the block $Q_{x}-$ $M_{1}$ in $\hat{Q}_{1}(\boldsymbol{z})$ (29), which is denoted as $\tilde{Q}_{1}(\boldsymbol{z})$. Using (30), we have

$$
\tilde{Q}_{1}(\boldsymbol{z}) \succeq Q_{1}^{*}(\boldsymbol{z}):=Q_{y}-M_{3}-\frac{1}{c_{2}} M_{2}^{\top} M_{2}
$$

We claim the following lemma to show $Q_{1}^{*}(\boldsymbol{z}) \succeq 0$, whose proof is provided in Appendix VI-C.

Lemma 6. When $\alpha$ is large enough and $\beta$ is positively small enough, $Q_{1}^{*}(\boldsymbol{z})$ in (31) is positive semidefinite, i.e. $Q_{1}^{*}(\boldsymbol{z}) \succeq 0$, for any $\boldsymbol{z} \in \mathbb{R}^{n+m+k}$.

In addition, to make the above Schur complement argument work, it requires that $c_{1}(\beta)>0$ and $c_{2}(\alpha, \beta)>0$. This condition can be achieved if we set parameter $\alpha$ large enough and $\beta$ positively small enough. Because for $\alpha, \beta>0, c_{2}(\alpha, \beta)$ is a scalar function that is strictly increasing in $\alpha$ and $c_{2} \rightarrow+\infty$ as $\alpha \rightarrow+\infty$ with fixed $\beta ; c_{1}(\beta)$ is a strictly decreasing function in $\beta$, and $c_{1} \rightarrow \frac{2 \eta_{x}}{\eta_{y}} \kappa_{1}>0$ as $\beta \rightarrow 0$.

According to the Schur complement theorem and lemma 6, we obtain $\tilde{Q}_{1}(\boldsymbol{z}) \succeq 0, \hat{Q}_{1}(\boldsymbol{z}) \succeq 0, \bar{Q}_{1}(\boldsymbol{z}) \succeq 0$ and eventually $Q_{1}(\boldsymbol{z}) \succeq 0$.

\footnotetext{
${ }^{1}$ For matrix $M=\left[\begin{array}{ll}A & B \\ C & D\end{array}\right]$, the Schur complement of the block $D$ in $M$ is defined as $M / D:=A-B D^{-1} C$.
}

\section{B. Proof of Lemma 5}

Since the proof of lemma 5 is very similar to the proof of lemma 3, without causing any confusion, we recycle the notations and definitions used in Appendix VI-A.

Plugging $P_{2}$ (23) into equation (25) with $\tau=\frac{\beta^{2}}{\alpha}$, we denote

$$
Q_{2}(\boldsymbol{z}):=-W(\boldsymbol{z})^{\top} P_{2}-P_{2} W(\boldsymbol{z})-\tau P_{2}
$$

The detailed formulation of $Q_{2}(\boldsymbol{z})$ is exactly the same as $Q_{1}(\boldsymbol{z})$ (26) except that the block $Q_{y}$ in $Q_{2}(\boldsymbol{z})$ is defined as

$$
Q_{y}^{\prime}:=2 \alpha G+2 \beta B^{\top} B-\frac{\beta^{2}}{\eta_{y}} I
$$

Here, we use notation $Q_{y}^{\prime}$ to distinguish with the corresponding block $Q_{y}$ in $Q_{1}(\boldsymbol{z})$ (26).

To prove Lemma 5, it is sufficient to show $Q_{2}(\boldsymbol{z}) \succeq 0$ for any $\boldsymbol{z} \in \mathbb{R}^{n+m+k}$, and we will use the Schur complement argument twice to prove it.

Firstly, consider the Schur complement of the block $Q_{\lambda}$ in $Q_{2}(\boldsymbol{z})(26)$, which is denoted as $\bar{Q}_{2}(\boldsymbol{z})$. Using (28), we have

$$
\bar{Q}_{2}(\boldsymbol{z}) \succeq \hat{Q}_{2}(\boldsymbol{z}):=\left[\begin{array}{cc}
Q_{x}-M_{1} & M_{2} \\
M_{2}^{\top} & Q_{y}^{\prime}-M_{3}
\end{array}\right]
$$

Here, $Q_{x}, M_{1}, M_{2}$ and $M_{3}$ have exactly the same definitions as those in (29).

Secondly, consider the Schur complement of the block $Q_{x}-$ $M_{1}$ in $\hat{Q}_{2}(\boldsymbol{z})$ (34), which is denoted as $\tilde{Q}_{2}(\boldsymbol{z})$. Using (30), we have

$$
\tilde{Q}_{2}(\boldsymbol{z}) \succeq Q_{2}^{*}(\boldsymbol{z}):=Q_{y}^{\prime}-M_{3}-\frac{1}{c_{2}} M_{2}^{\top} M_{2}
$$

By assumption 4 , we have

$$
2 \alpha G+2 \beta B^{\top} B \succeq 2 \beta\left(G+B^{\top} B\right) \succeq 2 \beta \gamma I
$$

and thus

$$
Q_{y}^{\prime}-M_{3} \succeq \beta \cdot \underbrace{\left[2 \gamma-\frac{\beta}{\eta_{y}}-\frac{\beta \omega}{c_{1} \eta_{\lambda}^{2}}\left(\frac{\beta^{2}}{\alpha}+\eta_{y} \rho\right)^{2}\right]}_{:=c_{3}(\alpha, \beta)} \cdot I
$$

Similar to lemma 6 , when set parameter $\alpha$ larger enough and $\beta$ positively small enough, we have $Q_{2}^{*}(\boldsymbol{z}) \succeq 0$ for any $z \in \mathbb{R}^{n+m+k}$. The proof sketch is that for sufficiently large $\alpha$ and sufficiently small $\beta,\left\|M_{2}^{\top} M_{2}\right\|$ is bounded. Since $c_{2}$ is dominated by $\alpha$, we can set $\alpha$ large enough such that $\frac{1}{c_{2}} \leq \beta^{2}$ for any fixed $\beta$, then select $\beta$ positively small enough such that $c_{3}-\beta\left\|M_{2}^{\top} M_{2}\right\|>0$. Thus we have $Q_{2}^{*}(\boldsymbol{z}) \succeq 0$.

Consequently, by the Schur complement theorem, we have $\tilde{Q}_{2}(\boldsymbol{z}) \succeq 0, \hat{Q}_{2}(\boldsymbol{z}) \succeq 0, \bar{Q}_{2}(\boldsymbol{z}) \succeq 0$ and eventually $Q_{2}(\boldsymbol{z}) \succeq 0$ for any $z \in \mathbb{R}^{n+m+k}$.

\section{Proof of Lemma 6}

As shown in equation (31), $Q_{1}^{*}(\boldsymbol{z})$ is defined as

$$
Q_{1}^{*}(\boldsymbol{z}):=Q_{y}-M_{3}-\frac{1}{c_{2}} M_{2}^{\top} M_{2}
$$

Consider the three items in $Q_{1}^{*}(\boldsymbol{z})$ one by one as follows.

For the first term, there exists a constant $\pi>0$ such that

$$
G+B^{\top} B=U\left(\Sigma+T T^{\top}\right) U^{\top} \succeq \pi \cdot U U^{\top}
$$


Because $\operatorname{ker}(G) \cap \operatorname{ker}(B)=\operatorname{span}\left(\boldsymbol{u}_{l+r+1}, \cdots, \boldsymbol{u}_{m}\right)$, we have $D:=\Sigma+T T^{\top} \succ 0$. Otherwise, if $D$ is just positive semidefinite, there exists $\boldsymbol{w} \in \mathbb{R}^{l+r} \neq \mathbf{0}$ such that $D \boldsymbol{w}=\mathbf{0}$. Then we find a vector $\boldsymbol{v}:=U \boldsymbol{w} \neq \mathbf{0}$ such that

$$
\boldsymbol{v}^{\top}\left(G+B^{\top} B\right) \boldsymbol{v}=\boldsymbol{v}^{\top} U D U^{\top} \boldsymbol{v}=\boldsymbol{w}^{\top} D \boldsymbol{w}=0
$$

Since $G$ and $B^{\top} B$ are both positive semi-definite, we have $\boldsymbol{v} \in \operatorname{ker}(G) \cap \operatorname{ker}(B)$. However, by the definition $\boldsymbol{v}:=$ $U \boldsymbol{w} \in \operatorname{span}\left(\boldsymbol{u}_{1}, \cdots, \boldsymbol{u}_{l+r}\right)$, which is contradictory. Thus $D$ is positive definite and $D \succeq \pi I$ where $\pi$ is the smallest eigenvalue of $D$. As a result, we have

$$
Q_{y}=2 \alpha G+2 \beta B^{\top} B-\frac{\beta^{2}}{\eta_{y}} U U^{\top} \succeq \beta \underbrace{\left(2 \pi-\beta / \eta_{y}\right)}_{:=h_{1}(\beta)} U U^{\top}
$$

For the second term, using $B=T^{\top} U^{\top}$ and $G=U \Sigma U^{\top}$, we obtain

$$
\begin{aligned}
M_{3}= & \frac{\beta^{2}}{c_{1} \eta_{\lambda}^{2}} U\left[\frac{\beta^{4}}{\alpha^{2}} T T^{\top}+\eta_{y}^{2} \Sigma T T^{\top} \Sigma\right. \\
& \left.\quad-\frac{\eta_{y} \beta^{2}}{\alpha}\left(\Sigma T T^{\top}+T T^{\top} \Sigma\right)\right] U^{\top} \\
& \preceq \beta \cdot h_{2}(\alpha, \beta) \cdot U U^{\top}
\end{aligned}
$$

where

$$
\begin{array}{r}
h_{2}(\alpha, \beta):=\frac{\beta}{c_{1} \eta_{\lambda}^{2}}\left(\frac{\beta^{4}}{\alpha^{2}}\left\|T T^{\top}\right\|+\eta_{y}^{2}\left\|\Sigma T T^{\top} \Sigma\right\|\right. \\
\left.+\frac{\eta_{y} \beta^{2}}{\alpha}\left\|\Sigma T T^{\top}+T T^{\top} \Sigma\right\|\right)
\end{array}
$$

For the third term, using $B=T^{\top} U^{\top}$ and $G=U \Sigma U^{\top}$, we have

$$
\begin{aligned}
M_{2}= & (\beta-1) A^{\top} B-\frac{\beta}{c_{1} \eta_{\lambda}^{2}}\left(\eta_{x} F-\frac{\beta^{2}}{\alpha} I\right) A^{\top} B\left(\frac{\beta^{2}}{\alpha} I-\eta_{y} G\right) \\
= & {\left[(\beta-1) A^{\top} T^{\top}\right.} \\
& \underbrace{\left.-\frac{\beta}{c_{1} \eta_{\lambda}^{2}}\left(\eta_{x} F-\frac{\beta^{2}}{\alpha} I\right) A^{\top}\left(\frac{\beta^{2}}{\alpha} T^{\top}-\eta_{y} B U \Sigma\right)\right]}_{:=H_{1}(\alpha, \beta)} \cdot U^{\top}
\end{aligned}
$$

and thus

$$
M_{2}^{\top} M_{2}=U \cdot H_{1}^{\top} H_{1} \cdot U^{\top} \preceq h_{3}(\alpha, \beta) \cdot U U^{\top}
$$

where

$$
\begin{aligned}
& h_{3}(\alpha, \beta):= \\
& {\left[\|T A\|+\frac{\beta}{c_{1} \eta_{\lambda}^{2}}\|A\|\left(\eta_{x} \ell+\frac{\beta^{2}}{\alpha}\right)\left(\frac{\beta^{2}}{\alpha}\|T\|+\eta_{y}\|B U \Sigma\|\right)\right]^{2}}
\end{aligned}
$$

Here, we use $|\beta-1| \leq 1$ since $0<\beta<1$.

In summary, we obtain

$$
\begin{aligned}
Q_{1}^{*}(\boldsymbol{z}) & =Q_{y}-M_{3}-\frac{1}{c_{2}} M_{4}^{\top} M_{4} \\
& \succeq \underbrace{\left[\beta\left(h_{1}(\beta)-h_{2}(\alpha, \beta)\right)-\frac{h_{3}(\alpha, \beta)}{c_{2}}\right]}_{:=h_{4}(\alpha, \beta)} \cdot U U^{\top}
\end{aligned}
$$

For sufficiently large $\alpha$ and sufficiently small $\beta, h_{3}$ is bounded. Since $c_{2}$ is dominated by $\alpha$, we can set $\alpha$ large enough such that $\frac{h_{3}}{c_{2}} \leq \beta^{2}$ for any fixed $\beta$, then select $\beta$ positively small enough such that $h_{1}-h_{2}-\beta>0$. Thus we have $h_{4}(\alpha, \beta)>0$ and $Q_{1}^{*}(\boldsymbol{z}) \succeq 0$ for any $\boldsymbol{z} \in \mathbb{R}^{n+m+k}$.

\section{Proof of theorem 3}

Let $H_{g}^{*}=\nabla^{2} g\left(\boldsymbol{y}^{*}\right)$ be the Hessian matrix of $g(\boldsymbol{y})$ at $\boldsymbol{y}^{*}$ and $D(\delta)=\left\{\boldsymbol{z} \mid\left\|\boldsymbol{z}-\boldsymbol{z}^{*}\right\| \leq \delta\right\}$ be the $\delta$-neighbor of $\boldsymbol{z}^{*}$. Using Taylor's expansion, we have

$$
\boldsymbol{\theta}(\boldsymbol{y}):=\nabla g(\boldsymbol{y})-\left(\nabla g\left(\boldsymbol{y}^{*}\right)+H_{g}^{*} \cdot \Delta \boldsymbol{y}\right) \sim O\left(\|\Delta \boldsymbol{y}\|^{2}\right)
$$

which means that there exist positive constants $m_{0}$ and $\delta$ such that we have $\|\boldsymbol{\theta}(\boldsymbol{y})\| \leq m_{0} \cdot\|\Delta \boldsymbol{y}\|^{2}$ for any $\boldsymbol{z} \in D(\delta)$. Since

$$
\frac{d \boldsymbol{z}}{d t}=\underbrace{\left[\begin{array}{ccc}
-\eta_{x} F(\boldsymbol{x}) & 0 & -\eta_{x} A^{\top} \\
0 & -\eta_{y} H_{g}^{*} & -\eta_{y} B^{\top} \\
\eta_{\lambda} A & \eta_{\lambda} B & 0
\end{array}\right]}_{:=W_{0}(\boldsymbol{x})} \Delta \boldsymbol{z}-\eta_{y} \underbrace{\left[\begin{array}{c}
0 \\
\boldsymbol{\theta}(\boldsymbol{y}) \\
0
\end{array}\right]}_{:=\hat{\boldsymbol{\theta}}(\boldsymbol{y})}
$$

we have

$$
\begin{aligned}
\frac{d V_{2}(\boldsymbol{z})}{d t}= & {\left[W_{0}(\boldsymbol{x}) \Delta \boldsymbol{z}-\eta_{y} \hat{\boldsymbol{\theta}}(\boldsymbol{y})\right]^{\top} P_{2} \Delta \boldsymbol{z} } \\
& +\Delta \boldsymbol{z}^{\top} P_{2}\left[W_{0}(\boldsymbol{x}) \Delta \boldsymbol{z}-\eta_{y} \hat{\boldsymbol{\theta}}(\boldsymbol{y})\right] \\
= & \Delta \boldsymbol{z}^{\top} W_{0}(\boldsymbol{x})^{\top} P_{2} \Delta \boldsymbol{z}+\Delta \boldsymbol{z}^{\top} P_{2} W_{0}(\boldsymbol{x}) \Delta \boldsymbol{z} \\
& -2 \alpha \Delta \boldsymbol{y}^{\top} \boldsymbol{\theta}(\boldsymbol{y})+2 \frac{\eta_{y}}{\eta_{\lambda}} \beta \Delta \boldsymbol{\lambda}^{\top} B \boldsymbol{\theta}(\boldsymbol{y})
\end{aligned}
$$

where $V_{2}(\boldsymbol{z})$ and $P_{2}$ are defined as (22) and (23) respectively.

By the proof of theorem 2 and condition (14), there exists $\tau_{0}>0$ such that for any $\boldsymbol{z} \in D(\delta)$

$$
\begin{aligned}
\frac{d V_{2}(\boldsymbol{z})}{d t} & \leq-\tau_{0} \Delta \boldsymbol{z}^{\top} P_{2} \Delta \boldsymbol{z}-2\left[\alpha \Delta \boldsymbol{y}^{\top}-\frac{\beta \eta_{y}}{\eta_{\lambda}} \Delta \boldsymbol{\lambda}^{\top} B\right] \boldsymbol{\theta}(\boldsymbol{y}) \\
& \leq-\tau_{0} \Delta \boldsymbol{z}^{\top} P_{2} \Delta \boldsymbol{z}+2 m_{0}\left(\alpha+\frac{\beta \eta_{y}}{\eta_{\lambda}}\|B\|\right)\|\Delta \boldsymbol{z}\|^{3} \\
& \leq-\frac{\tau_{0}}{2} \Delta \boldsymbol{z}^{\top} P_{2} \Delta \boldsymbol{z}-\left(\frac{\tau_{0}}{2} \sigma_{\min }-m_{1}\|\Delta \boldsymbol{z}\|\right)\|\Delta \boldsymbol{z}\|^{2}
\end{aligned}
$$

where $m_{1}:=2 m_{0}\left(\alpha+\frac{\beta \eta_{y}}{\eta_{\lambda}}\|B\|\right)$ and $\sigma_{\min }$ is the minimal eigenvalue of $P_{2}$.

Taking $\delta$ sufficiently small such that $\delta \leq \frac{\tau_{0}}{2 m_{1}} \sigma_{\min }$, we have

$$
\frac{d V_{2}(\boldsymbol{z})}{d t} \leq-\frac{\tau_{0}}{2} \Delta \boldsymbol{z}^{\top} P_{2} \Delta \boldsymbol{z}=-\frac{\tau_{0}}{2} V_{2}(\boldsymbol{z})
$$

Since $\boldsymbol{z}(t)$ asymptotically converges to $\boldsymbol{z}^{*}$, there exists a time $t_{\delta} \geq 0$ such that $\|\Delta \boldsymbol{z}\| \leq \delta$ for any $t \geq t_{\delta}$. Hence, theorem 3 is proved.

\section{E. Proof of Lemma 2}

We use the Schur complement argument twice to prove lemma 2. Firstly, consider the Schur complement of the block $\frac{\alpha}{\eta_{x}} I$ in matrix $P_{1}(17)$, which is denoted as $\hat{P}_{1}$

$$
\begin{aligned}
\hat{P}_{1} & =\left[\begin{array}{cc}
\frac{\alpha}{\eta_{y}} U U^{\top} & -\frac{\beta}{\eta_{\lambda}} B^{\top} \\
-\frac{\beta}{\eta_{\lambda}} B & \frac{\alpha}{\eta_{\lambda}} I-\frac{\eta_{x}}{\alpha \eta_{\lambda}^{2}} A A^{\top}
\end{array}\right] \\
& \succeq\left[\begin{array}{cc}
\frac{\alpha}{\eta_{y}} U U^{\top} & -\frac{\beta}{\eta_{\lambda}} B^{\top} \\
-\frac{\beta}{\eta_{\lambda}} B & s_{1}(\alpha) \cdot I
\end{array}\right]:=\bar{P}_{1}
\end{aligned}
$$


where $s_{1}(\alpha):=\frac{\alpha}{\eta_{\lambda}}-\frac{\eta_{x}}{\alpha \eta_{\lambda}^{2}} \kappa_{2}$. Then consider the Schur complement of the block $s_{1}(\alpha) \cdot I$ in matrix $\bar{P}_{1}$, which is

$$
\begin{aligned}
\tilde{P}_{1} & =\frac{\alpha}{\eta_{y}} U U^{\top}-\frac{1}{s_{1}(\alpha)} \cdot \frac{\beta^{2}}{\eta_{\lambda}^{2}} B^{\top} B \\
& =U \underbrace{\left[\frac{\alpha}{\eta_{y}} I-\frac{1}{s_{1}(\alpha)} \cdot \frac{\beta^{2}}{\eta_{\lambda}^{2}} T T^{\top}\right]}_{:=S_{1}(\alpha, \beta)} U^{\top}
\end{aligned}
$$

For $\alpha>0, s_{1}(\alpha)$ is strictly increasing in $\alpha$ and $s_{1}(\alpha) \rightarrow+\infty$ as $\alpha \rightarrow+\infty$. Hence, $S_{1}(\alpha, \beta) \succ 0$ when we set parameter $\alpha$ large enough and $\beta$ positively small enough. By the Schur complement theorem, we have $\tilde{P}_{1} \succeq 0, \bar{P}_{1} \succeq 0, \hat{P}_{2} \succeq 0$ and eventually $P_{1} \succeq 0$.

Denote $\Delta \boldsymbol{x}=\boldsymbol{x}-\boldsymbol{x}^{*}, \Delta \boldsymbol{y}=\boldsymbol{y}-\boldsymbol{y}^{*}$ and $\Delta \boldsymbol{\lambda}=\boldsymbol{\lambda}-\boldsymbol{\lambda}^{*}$. Then for the Lyapunov function $V_{1}(\boldsymbol{z})$ (16), we have

$$
\begin{aligned}
V_{1}(\boldsymbol{z})=\frac{\alpha}{\eta_{x}} & \|\Delta \boldsymbol{x}\|^{2}+\frac{\alpha}{\eta_{y}}\left\|U^{\top} \Delta \boldsymbol{y}\right\|^{2}+\frac{\alpha}{\eta_{\lambda}}\|\Delta \boldsymbol{\lambda}\|^{2} \\
& +\frac{2}{\eta_{\lambda}} \Delta \boldsymbol{x}^{\top} A^{\top} \Delta \boldsymbol{\lambda}-\frac{2 \beta}{\eta_{\lambda}} \Delta \boldsymbol{y}^{\top} B^{\top} \Delta \boldsymbol{\lambda} \\
=\| \frac{1}{\eta_{\lambda}} & A \Delta \boldsymbol{x}+\Delta \boldsymbol{\lambda}\left\|^{2}+\right\| \frac{\beta}{\eta_{\lambda}} U^{\top} \Delta \boldsymbol{y}-T \Delta \boldsymbol{\lambda} \|^{2} \\
& +\Delta \boldsymbol{x}^{\top}\left(\frac{\alpha}{\eta_{x}} I-\frac{1}{\eta_{\lambda}^{2}} A^{\top} A\right) \Delta \boldsymbol{x} \\
& +\left(\frac{\alpha}{\eta_{y}}-\frac{\beta^{2}}{\eta_{\lambda}^{2}}\right)\left\|U^{\top} \Delta \boldsymbol{y}\right\|^{2} \\
& +\Delta \boldsymbol{\lambda}^{\top}\left(\left(\frac{\alpha}{\eta_{y}}-1\right) I-T^{\top} T\right) \Delta \boldsymbol{\lambda}
\end{aligned}
$$

Set parameter $\alpha$ sufficiently large and $\beta$ positively small enough such that

$$
\alpha / \eta_{x}>\kappa_{2} / \eta_{\lambda}^{2}, \quad \alpha / \eta_{y}>\beta^{2} / \eta_{\lambda}^{2}, \quad \alpha / \eta_{\lambda}-1>\left\|T^{\top} T\right\|,
$$

Then $V_{1}(\boldsymbol{z})=0 \Longleftrightarrow \Delta \boldsymbol{x}=\mathbf{0}, \Delta \boldsymbol{\lambda}=\mathbf{0}, U^{\top} \Delta \boldsymbol{y}=\mathbf{0}$ and

$$
U^{\top} \Delta \boldsymbol{y}=\mathbf{0} \Longleftrightarrow B \Delta \boldsymbol{y}=\mathbf{0}, G \Delta \boldsymbol{y}=\mathbf{0}
$$

due to $\operatorname{ker}(G) \cap \operatorname{ker}(B)=\operatorname{span}\left(\boldsymbol{u}_{l+r+1}, \cdots, \boldsymbol{u}_{m}\right)$.

\section{F. Proof of Lemma 4}

Consider the Schur complement of the block $\frac{\alpha}{\eta_{x}} I$ in matrix $P_{2}$ (23), which is

$$
\hat{P}_{2}=\left[\begin{array}{cc}
\frac{\alpha}{\eta_{y}} I & -\frac{\beta}{\eta_{\lambda}} B^{\top} \\
-\frac{\beta}{\eta_{\lambda}} B & \frac{\alpha}{\eta_{\lambda}} I-\frac{\eta_{x}}{\alpha \eta_{\lambda}^{2}} A A^{\top}
\end{array}\right]
$$

Then consider the Schur complement of the block $\frac{\alpha}{\eta_{y}} I$ in matrix $\hat{P}_{2}$, which is

$$
\begin{aligned}
\bar{P}_{2} & =\frac{\alpha}{\eta_{\lambda}} I-\frac{\eta_{x}}{\alpha \eta_{\lambda}^{2}} A A^{\top}-\frac{\eta_{y} \beta^{2}}{\alpha \eta_{\lambda}^{2}} B B^{\top} \\
& =\underbrace{\left(\frac{\alpha}{\eta_{\lambda}}-\frac{\eta_{x}}{\alpha \eta_{\lambda}^{2}} \kappa_{2}-\frac{\eta_{y} \beta^{2}}{\alpha \eta_{\lambda}^{2}}\left\|B B^{\top}\right\|\right)}_{p_{1}(\alpha, \beta)} \cdot I
\end{aligned}
$$

It is easy to check that $p_{1}(\alpha, \beta)>0$ when we set parameter $\alpha$ large enough and $\beta$ positively small enough, thus $\bar{P}_{2} \succ$ 0 . By the Schur complement theorem, we have $\hat{P}_{2} \succ 0$ and eventually $P_{2} \succ 0$.

Since $P_{2} \succ 0$, we have $V_{2}(\boldsymbol{z})=0 \Longleftrightarrow \boldsymbol{z}=\boldsymbol{z}^{*}$.

\section{G. Proof of Proposition 3 and Proposition 4}

Let $\hat{\boldsymbol{z}}:=\left[\hat{\boldsymbol{x}}^{\top}, \hat{\boldsymbol{y}}^{\top}, \hat{\boldsymbol{\lambda}}^{\top}\right]^{\top}$ be another equilibrium point of PDGD (3). By the definition, we have

$$
\begin{aligned}
\nabla f(\hat{\boldsymbol{x}})-\nabla f\left(\boldsymbol{x}^{*}\right) & =-A^{\top}\left(\hat{\boldsymbol{\lambda}}-\boldsymbol{\lambda}^{*}\right) \\
\nabla g(\hat{\boldsymbol{y}})-\nabla g\left(\boldsymbol{y}^{*}\right) & =-B^{\top}\left(\hat{\boldsymbol{\lambda}}-\boldsymbol{\lambda}^{*}\right) \\
\mathbf{0} & =A\left(\hat{\boldsymbol{x}}-\boldsymbol{x}^{*}\right)+B\left(\hat{\boldsymbol{y}}-\boldsymbol{y}^{*}\right)
\end{aligned}
$$

Multiply $\left(\hat{\boldsymbol{x}}-\boldsymbol{x}^{*}\right)^{\top},\left(\hat{\boldsymbol{y}}-\boldsymbol{y}^{*}\right)^{\top}$ and $\left(\hat{\boldsymbol{\lambda}}-\boldsymbol{\lambda}^{*}\right)^{\top}$ to the both sides of (37a), (37b) and (37c) respectively and sum them up, we obtain

$$
\begin{aligned}
(\nabla f(\hat{\boldsymbol{x}}) & \left.-\nabla f\left(\boldsymbol{x}^{*}\right)\right)^{\top}\left(\hat{\boldsymbol{x}}-\boldsymbol{x}^{*}\right) \\
& +\left(\nabla g(\hat{\boldsymbol{y}})-\nabla g\left(\boldsymbol{y}^{*}\right)\right)^{\top}\left(\hat{\boldsymbol{y}}-\boldsymbol{y}^{*}\right)=\mathbf{0}
\end{aligned}
$$

Due to the strong convexity of $f(\boldsymbol{x})$ and convexity of $g(\boldsymbol{y})$, we have

$$
\hat{\boldsymbol{x}}=\boldsymbol{x}^{*},\left(g(\hat{\boldsymbol{y}})-\nabla g\left(\boldsymbol{y}^{*}\right)\right)^{\top}\left(\hat{\boldsymbol{y}}-\boldsymbol{y}^{*}\right)=\mathbf{0}
$$

Since $A$ is of full row rank (assumption 3), we have $\hat{\lambda}=\lambda^{*}$ due to (37a) and (38). By (37c), we obtain $B \hat{\boldsymbol{y}}=B \boldsymbol{y}^{*}$. By (37b), we have $\nabla g(\hat{\boldsymbol{y}})=\nabla g\left(\boldsymbol{y}^{*}\right)$, which is $G \hat{\boldsymbol{y}}=G \boldsymbol{y}^{*}$ in (10). Hence, proposition 3 is proved.

For proposition 4 , by assumption 4 we further have

$$
\left\|\hat{\boldsymbol{y}}-\boldsymbol{y}^{*}\right\|^{2} \leq 0 \Longrightarrow \hat{\boldsymbol{y}}=\boldsymbol{y}^{*}
$$

Hence, the equilibrium point $\left(\boldsymbol{x}^{*}, \boldsymbol{\lambda}^{*}, \boldsymbol{y}^{*}\right)$ is unique.

\section{H. Proof of Proposition 2}

From (6a), we have

$$
\begin{aligned}
& \boldsymbol{x}_{i+1}-\boldsymbol{x}^{*}=\boldsymbol{x}_{i}-\boldsymbol{x}^{*} \\
& -\nu_{x}\left(\nabla f\left(\boldsymbol{x}_{i}\right)-\nabla f\left(\boldsymbol{x}^{*}\right)+A^{\top}\left(\boldsymbol{\lambda}_{i}-\boldsymbol{\lambda}^{*}\right)\right) \\
& \Longrightarrow\left\|A^{\top}\left(\boldsymbol{\lambda}_{i}-\boldsymbol{\lambda}^{*}\right)\right\| \leq\left\|\nabla f\left(\boldsymbol{x}_{i}\right)-\nabla f\left(\boldsymbol{x}^{*}\right)\right\| \\
& +\frac{1}{\nu_{x}}\left(\left\|\boldsymbol{x}_{i}-\boldsymbol{x}^{*}\right\|+\left\|\boldsymbol{x}_{i+1}-\boldsymbol{x}^{*}\right\|\right) \\
& \leq\left(\ell+\frac{2}{\nu_{x}}\right)\left\|\boldsymbol{x}_{i}-\boldsymbol{x}^{*}\right\| \\
& \Longrightarrow\left\|\boldsymbol{\lambda}_{i}-\boldsymbol{\lambda}^{*}\right\| \leq \frac{\ell+2 / \nu_{x}}{\sqrt{\kappa_{1}}} \cdot\left\|\boldsymbol{x}_{i}-\boldsymbol{x}^{*}\right\| \leq c_{\lambda} \cdot \vartheta^{i}
\end{aligned}
$$

where $c_{\lambda}:=\frac{c_{x}}{\sqrt{\kappa_{1}}}\left(\ell+\frac{2}{\nu_{x}}\right)$.

Similarly, from (6c), we have

$$
\begin{aligned}
& \left\|B\left(\boldsymbol{y}_{i}-\boldsymbol{y}^{*}\right)\right\| \leq\left\|A\left(\boldsymbol{x}_{i}-\boldsymbol{x}^{*}\right)\right\| \\
& \quad+\frac{1}{\nu_{\lambda}}\left(\left\|\boldsymbol{\lambda}_{i+1}-\boldsymbol{\lambda}^{*}\right\|+\left\|\boldsymbol{\lambda}_{i}-\boldsymbol{\lambda}^{*}\right\|\right) \\
& \leq\|A\| \cdot\left\|\boldsymbol{x}_{i}-\boldsymbol{x}^{*}\right\|+\frac{2}{\nu_{\lambda}}\left\|\boldsymbol{\lambda}_{i}-\boldsymbol{\lambda}^{*}\right\| \leq c_{y} \cdot \vartheta^{i}
\end{aligned}
$$

where $c_{y}:=c_{x} \cdot\|A\|+\frac{2 c_{\lambda}}{\nu_{\lambda}}$.

From (6b), we have

$$
\begin{array}{r}
\left\|B\left(\nabla g\left(\boldsymbol{y}_{i}\right)-\nabla g\left(\boldsymbol{y}^{*}\right)\right)\right\| \leq\left\|B B^{\top}\right\| \cdot\left\|\boldsymbol{\lambda}_{i}-\boldsymbol{\lambda}^{*}\right\| \\
+\frac{2}{\nu_{y}}\left\|B\left(\boldsymbol{y}_{i}-\boldsymbol{y}^{*}\right)\right\| \leq c_{g} \cdot \vartheta^{i}
\end{array}
$$

where $c_{g}:=c_{\lambda} \cdot\left\|B B^{\top}\right\|+\frac{2 c_{y}}{\nu_{y}}$. 